International Mathematical Forum, 1, 2006, no. 9, 433-443

\title{
A computing method for the Moore-Penrose inverse of some special matrices
}

\author{
Sadullah Sakallığlu and Fikri Akdeniz \\ Department of Statistics, Faculty of Art and Sciences \\ University of Çukurova, 01330 Adana, Turkey \\ sadullah@mail.cu.edu.tr \\ akdeniz@mail.cu.edu.tr
}

\begin{abstract}
A number of methods have been proposed for computing the Moore Penrose (M-P) inverse, $X^{+}$, of an arbitrary mxn matrix of rank $r \leq m \leq$ $n$. In this paper we have given a simple procedure to find the explicit form of M-P inverse of a matrix that has special form and will consider the ANOVA models. Also computational algorithms for constructing and computing M-P inverse of these special matrices are given.
\end{abstract}

Keywords: Direct (Kronecker) Product; Factorial Design; Moore - Penrose Inverse.

Mathematics Subject Classification: 15A09, 62K10.

\section{Introduction}

The application of generalized inverse matrices to linear statistical models has been of considerable interest since early 1960's. Generalized inverse of a matrix plays significant role in mathematical statistics, also Moore-Penrose (M-P) inverse. The M-P inverse of a matrix is the basis for much of the modern development in regression analysis and analysis of variance. For an extensive discussion of the theory of M-P inverses, we refer, e.g. to the books by Boullion and Odell [3], Pringle and Rayner [8], Rao and Mitra [9], Albert [1], Ben Israel and Greville [2], Searle [10], Graybill [4]. Many statisticians contributed to the derivation of the M-P inverse $X^{+}$for the matrices appearing in special statistical models. But it is not easy to compute, especially when has many columns. Most matrices occurring in the linear model can be represented as 
direct products of identity matrices and matrices with all elements equal to 1 . Using this regularity, Kim ad Lee [5] provided general explicit forms $X^{+}$for the classificatory models. Sunwoo [12] described an algorithm for computing sums of square without storing the design matrix. He also showed that such matrices can be obtained by a simple rule.

In this paper we shall give a simple procedure to compute the M-P inverse of a matrix that has a special form. This method can be applied to one, and multi - way factorial designs. We will also derive computational algorithms for constructing direct (Kronecker) product and computing the M-P inverse of matrix that has a special form.

\section{The Moore - Penrose Inverse and Background Information}

Definition 2.1 (Searle [10], p.212, Schott [11], p.171) The $n x m$ matrix $A^{+}$is called M-P inverse of $m x n$ matrix $A$ if satisfies the following four conditions:

i) $A A^{+} A=A$, ii) $A^{+} A A+=A^{+}$, iii) $A A^{+}$is symmetric, iv) $A^{+} A$ is symmetric.

If a matrix is satisfied only condition (i) it is called a generalized inverse.

Theorem 2.1 (Graybill [4], p.112). If $A$ is an $m x n$ matrix, then $A^{+}=$ $A^{\prime}\left(A A^{\prime}\right)^{+}=\left(A^{\prime} A\right) A^{\prime}$.

Definition 2.2 Let $A$ be an $m_{1} x n_{1}$ matrix and let $B$ be an $m_{2} x n_{2}$ matrix then the direct product of $A$ and $B$, which we write $A \otimes B$, is a matrix $C$ of size $m_{1} m_{2} x n_{1} n_{2}$ defined by $C=\left[a_{i j} B\right], i=1,2, \ldots, m_{1}, j=1,2, \ldots, n_{1}$.

Actually the definition is a right direct product (see Graybill [4], Harville [5]). Direct product has many useful properties some of which are as follows:

(1) $(A \otimes B)^{\prime}=A^{\prime} \otimes B^{\prime}$

$(2 \mathrm{i})(A \otimes B)^{-1}=A^{-1} \otimes B^{-1},(2 \mathrm{ii})(A \otimes B)^{+}=A^{+} \otimes B^{+}$

(3) $(A \otimes B)(C \otimes D)=A C \otimes B D$

(4) $(A+B) \otimes C=(A \otimes C)+B \otimes C$.

Theorem 2.2 (Graybill [4], p.115). If $A=A_{1}+A_{2}+\ldots+A_{t}$ and $A_{i} A_{j}^{\prime}=\emptyset$, $A_{i}^{\prime} A_{j}=\emptyset$ for all $i, j=1,2, \ldots, t, i \neq j$. Then $A^{+}=A_{1}^{+}+A_{2}^{+}+\ldots+A_{t}^{+}$. 


\section{Main Results}

Theorem 3.1 Suppose that the matrix $X$ can be partitioned into sub-matrices $X=\left[X_{0}, X_{1}, X_{2}, \ldots, X_{m}\right]$, where $X_{i}=j_{n_{1}} \otimes j_{n_{2}} \otimes \ldots \otimes I_{n_{i}} \otimes \ldots \otimes j_{n_{k-1}} \otimes j_{n_{k}}$ for $i=1,2, \ldots, m, m \leq k$, that is each consist of the direct product of $\mathrm{k}$ matrices such that $i^{\text {th }}$ matrix is identity matrix other ones are vectors with all elements equal to 1 , and $X_{0}=j_{N}=j_{n_{1}} \otimes j_{n_{2}} \otimes \ldots \otimes j_{n_{k-1}} \otimes j_{n_{k}}$ then the M-P inverse of the matrix $X$ is given by

$$
X^{+}=\left[\begin{array}{c}
\frac{a}{N} j_{n_{1}} \otimes j_{n_{2}}^{\prime} \otimes \ldots \otimes j_{n_{k-1}}^{\prime} \otimes j_{n_{k}}^{\prime} \\
\frac{n_{1}}{N} X_{1}^{\prime}+\left(\frac{a}{n_{1}}-1\right) \frac{1}{N} J_{n_{1}} \otimes j_{n_{2}}^{\prime} \otimes \ldots \otimes j_{n_{m}}^{\prime} \otimes \ldots \otimes j_{n_{k}}^{\prime} \\
\cdot \\
\cdot \\
\frac{n_{m}}{N} X_{m}^{\prime}+\left(\frac{a}{n_{m}}-1\right) \frac{1}{N} J_{n_{1}} \otimes j_{n_{2}}^{\prime} \otimes \ldots \otimes J_{n_{m}} \otimes \ldots \otimes j_{n_{k}}^{\prime}
\end{array}\right]
$$

where $a=\frac{1}{\sum_{i=0}^{m} \frac{1}{n_{i}}}, n_{0}=1, N=\prod_{i=1}^{k} n_{i}, j_{p}$ is the $p x 1$ vector of ones, $J_{p}=j_{p} \otimes j_{p}^{\prime}$ is the $p x p$ matrix of ones, and $j_{p}^{\prime}$ is the transpose of $j_{p}$.

Proof : $X X^{\prime}=J_{n_{1}} \otimes J_{n_{2}} \otimes \ldots \otimes J_{n_{k}}+\sum_{i=0}^{m} J_{n_{1}} \otimes J_{n_{2}} \otimes \ldots \otimes I_{n_{i}} \otimes \ldots \otimes J_{n_{k}}$ If we write $X X^{\prime}=\left(\sum_{i=0}^{m} \frac{1}{n_{i}}\right) J_{n_{1}} \otimes J_{n_{2}} \otimes \ldots \otimes J_{n_{k}}+\sum_{i=0}^{m} J_{n_{1}} \otimes J_{n_{2}} \otimes \ldots \otimes\left(I_{n_{i}}-\frac{1}{n_{i}} J_{n_{i}}\right) \otimes \ldots \otimes J_{n_{k}}$, the matrix $X X^{\prime}$ satisfies the conditions in Theorem 2.2. So we get $X X^{\prime}=$ $\frac{a}{N^{2}} J_{n_{1}} \otimes J_{n_{2}} \otimes \ldots \otimes J_{n_{k}}+\frac{1}{N^{2}} \sum^{m} n_{i}^{2} J_{n_{1}} \otimes J_{n_{2}} \otimes \ldots \otimes\left(I_{n_{i}}-\frac{1}{n_{i}} J_{n_{i}}\right) \otimes \ldots \otimes J_{n_{k}}$ Then, using Theorem 2.1 we get equation (3.1). This completes the proof.

Theorem 3.2 Let $X=\left[X_{0}, X_{1}, X_{2}, \ldots, X_{k}\right]$ be partitioning of $X$ into $k+1$ sub-matrices, where sub-matrices are as follows:

$X_{i}$ is a block matrix consist of $\left(\begin{array}{c}k \\ i\end{array}\right)$ matrices, where $i=1,2, \ldots, k$. In each block of $X_{i}$ is the direct product of $i I_{n_{j}}$ identity matrices and $(k-i)$ $j_{n_{j}}$ vectors $(j=1,2, \ldots, k)$. Then the M-P inverse of the partitioned matrix is given by 


$$
X^{+}=\left[\begin{array}{c}
X_{0}^{\prime}\left(X X^{\prime}\right)^{+} \\
X_{1}^{\prime}\left(X X^{\prime}\right)^{+} \\
\cdot \\
\cdot \\
X_{k}^{\prime}\left(X X^{\prime}\right)^{+}
\end{array}\right]=\prod_{i=1}^{k} \frac{1}{n_{i}+1}\left[\begin{array}{c}
\tilde{X}_{0} \\
\tilde{X}_{1} \\
\tilde{X}_{2} \\
\cdot \\
\cdot \\
\tilde{X}_{k-1} \\
\tilde{X}_{k}
\end{array}\right]
$$

where $\tilde{X}_{i}=X_{i}^{\prime}\left(X X^{\prime}\right)^{+}$for $i=0,1,2, \ldots, k$ and $X^{+}$is a block matrix of $k+1$ sub-matrices. Here each $\tilde{X}_{i}$ is the transpose of the matrix obtained from $X_{i}$ by replacing (in each block) $I_{n_{j}}$ by $\left[\left(n_{j}+1\right) I_{n_{j}}-J_{n_{j}}\right]$.

Proof: It is obvious that matrix $X$ has $2^{k}$ block matrices. After the calculation we obtain

$$
\begin{aligned}
X X^{\prime}= & \left(I_{n_{1}}+J_{n_{1}}\right) \otimes\left(I_{n_{2}}+J_{n_{2}}\right) \otimes \ldots \otimes\left(I_{n_{k}}+J_{n_{k}}\right) \\
= & \left(1+\frac{1}{n_{1}}\right) J_{n_{1}} \otimes\left(I_{n_{2}}+J_{n_{2}}\right) \otimes \ldots \otimes\left(I_{n_{k}}+J_{n_{k}}\right)+ \\
& \left(I_{n_{1}}-\frac{1}{n_{1}}\right) J_{n_{1}} \otimes\left(I_{n_{2}}+J_{n_{2}}\right) \otimes \ldots \otimes\left(I_{n_{k}}+J_{n_{k}}\right)
\end{aligned}
$$

The matrix $X X^{\prime}$ satisfies the conditions in Theorem 2.1. Hence we get

$$
\begin{aligned}
\left(X X^{\prime}\right)^{+}= & \left.\frac{1}{n_{1}\left(n_{1}+1\right)} J_{n_{1}}\right) \otimes\left(I_{n_{2}}-\frac{1}{n_{2}+1} J_{n_{2}}\right) \otimes \ldots \otimes\left(I_{n_{k}}-\frac{1}{n_{k}+1} J_{n_{k}}\right)+ \\
& \left(I_{n_{1}}-\frac{1}{n_{1}}\right) J_{n_{1}} \otimes\left(I_{n_{2}}-\frac{1}{n_{2}+1} J_{n_{2}}\right) \otimes \ldots \otimes\left(I_{n_{k}}-\frac{1}{n_{k}+1} J_{n_{k}}\right)
\end{aligned}
$$

where $\left(I_{n_{i}}+J_{n_{i}}\right)^{-1}=I_{n_{i}}-\frac{1}{n_{i}+1} J_{n_{i}}$. Thus, using Theorem 2.1 we get equation (3.2). This completes the proof.

For example, second block of $X$ is

$$
X_{1}=\left[I_{n_{1}} \otimes j_{n_{2}} \otimes \ldots \otimes j_{n_{k}}, j_{n_{1}} \otimes I_{n_{2}} \otimes \ldots \otimes j_{n_{k}}, \ldots, j_{n_{1}} \otimes j_{n_{2}} \otimes \ldots \otimes I_{n_{k}}\right]
$$

and $X_{1}$ has $\left(\begin{array}{c}k \\ i\end{array}\right)=k$ sub-matrices. Using Theorem 3.2, we get

$$
\tilde{X}_{1}=X_{1}^{\prime}\left(X X^{\prime}\right)^{+}=\left[\begin{array}{c}
{\left[\left(n_{1}+1\right) I_{n_{1}}-J_{n_{1}}\right] \otimes j_{n_{2}}^{\prime} \otimes \ldots \otimes j_{n_{k}}^{\prime}} \\
j_{n_{1}}^{\prime} \otimes\left[\left(n_{2}+1\right) I_{n_{2}}-J_{n_{2}}\right] \otimes j_{n_{3}} \otimes \ldots \otimes j_{n_{k}}^{\prime} \\
\cdot \\
\cdot \\
j_{n_{1}}^{\prime} \otimes j_{n_{2}}^{\prime} \otimes \ldots \otimes j_{n_{k-1}}^{\prime} \otimes\left[\left(n_{k}+1\right) I_{n_{k}}-J_{n_{k}}\right]
\end{array}\right]
$$


Note that $\tilde{X}_{1}$ is the transpose of the matrix obtained from $X_{1}$ by replacing $I_{n_{j}}$ by $\left[\left(n_{j}+1\right) I_{n_{j}}-J_{n_{j}}\right]$ for $j=1,2, \ldots, k$.

Theorem 3.1 can be applied to find the M-P inverse of the design matrix of one-way classification model, and multi-way factorial designs with no interactions. For the multi-way factorial designs with interactions Theorem 3.2 can be applied. We will consider the three-way classification model without interaction and one observation per cell, and three-way classification with interactions and one observation per cell as examples.

Example 1 Consider the three-way classification model without interaction and one observation per cell

$$
y_{i j k}=\mu+\alpha_{i}+\beta_{j}+\gamma_{k}+\varepsilon_{i j k}
$$

where $i=1,2, \ldots, n_{1} ; j=1,2, \ldots, n_{2} ; k=1,2, \ldots, n_{3}, y_{i j k}$ is the response for the treatment when factor $A$ is at level $i^{\text {th }}$, factor $B$ is at the $j^{\text {th }}$ level, and factor $C$ is at the $k^{\text {th }}$ level. $\mu$ is the general mean, $\alpha_{i}$ is the effect on the response of the level of factor $A, \beta_{j}$ is the effect on the response of the level of factor $B, \gamma_{k}$ is the effect on the response of the level factor $C$ and $\varepsilon_{i j k}$ is the error term. In matrix form Equation (3.3) can be written as

$$
Y=X \beta+e
$$

where $Y^{\prime}=\left(y_{111}, y_{112}, \ldots, y_{11 n_{3}}, y_{121}, y_{122}, \ldots, y_{12 n_{3}}, \ldots, y_{1 n_{2} 1}, y_{1 n_{2} 2}, \ldots, y_{1 n_{2} n_{3}}, \ldots\right.$, $\left.y_{n_{1} n_{2} 1}, \ldots, y_{n_{1} n_{2} n_{3}}\right), \beta^{\prime}=\left(\mu, \alpha_{1}, \ldots, \alpha_{n_{1}}, \beta_{1}, \ldots, \beta_{n_{2}}, \gamma_{1}, \ldots, \gamma_{n_{3}}\right)$,

$X=\left(X_{0}, X_{1}, X_{2}, X_{3}\right), X_{0}=j_{n_{1}} \otimes j_{n_{2}} \otimes j_{n_{3}}, X_{1}=I_{n_{1}} \otimes j_{n_{2}} \otimes j_{n_{3}}$, $X_{2}=j_{n_{1}} \otimes I_{n_{2}} \otimes j_{n_{3}}$ and $X_{3}=j_{n_{1}} \otimes j_{n_{2}} \otimes I_{n_{3}}$. If we take $k=3$ and $m=3$ in Theorem 3.1 we get

$$
X=\left(j_{n_{1}} \otimes j_{n_{2}} \otimes j_{n_{3}}, I_{n_{1}} \otimes j_{n_{2}} \otimes j_{n_{3}}, j_{n_{1}} \otimes I_{n_{2}} \otimes j_{n_{3}}, j_{n_{1}} \otimes j_{n_{2}} \otimes I_{n_{3}}\right) .
$$

So we have a design matrix for three-way classification without interaction and one observation per cell. Thus, from Theorem 2.1 we obtain the M-P inverse of $X$

$$
X^{+}=\left[\begin{array}{c}
\frac{a}{N} j_{n_{1}}^{\prime} \otimes j_{n_{2}}^{\prime} \otimes j_{n_{3}}^{\prime} \\
{\left[I_{n_{1}}+\left(\frac{a}{n_{1}}-1\right) J_{n_{1}}\right] \otimes \frac{1}{n_{2}} j_{n_{2}}^{\prime} \otimes \frac{1}{n_{3}} j_{n_{3}}^{\prime}} \\
\frac{1}{n_{1}} j_{n_{1}}^{\prime} \otimes\left[I_{n_{2}}+\left(\frac{a}{n_{2}}-1\right) J_{n_{2}}\right] \otimes \frac{1}{n_{3}} j_{n_{3}}^{\prime} \\
\frac{1}{n_{1}} j_{n_{1}}^{\prime} \otimes \frac{1}{n_{2}} j_{n_{2}}^{\prime} \otimes\left[I_{n_{3}}+\left(\frac{a}{n_{3}}-1\right) J_{n_{3}}\right]
\end{array}\right]
$$

where $a=\frac{n_{1} n_{2} n_{3}}{n_{1} n_{2} n_{3}+n_{1} n_{2}+n_{1} n_{3}+n_{2} n_{3}}$ and $N=n_{1} n_{2} n_{3}$.

Example 2 Consider the following three way classification model with interaction and one observation per cell

$$
y_{i j k}=\mu+\alpha_{i}+\beta_{j}+\gamma_{k}+(\alpha \beta)_{i j}+(\alpha \gamma)_{i k}+(\beta \gamma)_{j k}+(\alpha \beta \gamma)_{i j k}+\varepsilon_{i j k}
$$


where $i=1,2, \ldots, n_{1} ; j=1,2, \ldots, n_{2} ; k=1,2, \ldots, n_{3}$. In Theorem 3.2 if we take $k=3$ we have the related design matrix, as $X=\left(X_{0}, X_{1}, X_{2}, X_{3}\right)$, where $X_{0}=\left(j_{n_{1}} \otimes j_{n_{2}} \otimes j_{n_{3}}\right), X_{1}=\left(I_{n_{1}} \otimes j_{n_{2}} \otimes j_{n_{3}}, j_{n_{1}} \otimes I_{n_{2}} \otimes j_{n_{3}}, j_{n_{1}} \otimes j_{n_{2}} \otimes I_{n_{3}}\right)$, $X_{2}=\left(I_{n_{1}} \otimes I_{n_{2}} \otimes j_{n_{3}}, I_{n_{1}} \otimes j_{n_{2}} \otimes I_{n_{3}}, j_{n_{1}} \otimes I_{n_{2}} \otimes I_{n_{3}}\right)$ and $X_{3}=\left(I_{n_{1}} \otimes I_{n_{2}} \otimes I_{n_{3}}\right)$.

Using the Theorem 3.2, we obtain the $M-P$ inverse of as

$$
X^{+}=u\left[\begin{array}{c}
j_{n_{1}}^{\prime} \otimes j_{n_{2}}^{\prime} \otimes j_{n_{3}}^{\prime} \\
{\left[\left(n_{1}+1\right) I_{n_{1}}-J_{n_{1}}\right] \otimes j_{n_{2}}^{\prime} \otimes j_{n_{3}}^{\prime}} \\
j_{n_{1}}^{\prime} \otimes\left[\left(n_{2}+1\right) I_{n_{2}}-J_{n_{2}}\right] \otimes j_{n_{3}}^{\prime} \\
j_{n_{1}}^{\prime} \otimes j_{n_{2}}^{\prime} \otimes\left[\left(n_{3}+1\right) I_{n_{3}}-J_{n_{3}}\right] \\
{\left[\left(n_{1}+1\right) I_{n_{1}}-J_{n_{1}}\right] \otimes\left[\left(n_{2}+1\right) I_{n_{2}}-J_{n_{2}}\right] \otimes j_{n_{3}}^{\prime}} \\
{\left[\left(n_{1}+1\right) I_{n_{1}}-J_{n_{1}}\right] \otimes j_{n_{2}}^{\prime} \otimes\left[\left(n_{3}+1\right) I_{n_{3}}-J_{n_{3}}\right]} \\
j_{n_{1}}^{\prime} \otimes\left[\left(n_{2}+1\right) I_{n_{2}}-J_{n_{2}}\right] \otimes\left[\left(n_{3}+1\right) I_{n_{3}}-J_{n_{3}}\right] \\
{\left[\left(n_{1}+1\right) I_{n_{1}}-J_{n_{1}}\right] \otimes\left[\left(n_{2}+1\right) I_{n_{2}}-J_{n_{2}}\right] \otimes\left[\left(n_{3}+1\right) I_{n_{3}}-J_{n_{3}}\right]}
\end{array}\right]
$$

where $u=\prod_{i=1}^{3} \frac{1}{n_{i}+1}$

\section{Algorithms}

Let $\mathrm{P}$ a matrix defined to be of the form

$$
P=A_{1} \otimes A_{2} \otimes \ldots \otimes A_{k}
$$

where each matrix $A_{i}$ is either $I_{n_{i}}$ or $j_{n_{i}}$. Each matrix $X_{j}$ in Theorem 3.1 is in the form of (4.1). For the convenience of description of the algorithm define

$$
a_{i}=\left\{\begin{array}{l}
1 \text { if } A_{i}=I_{n_{i}} \\
0 \text { if } A_{i}=j_{n_{i}}
\end{array} \text { for } i=1,2, \ldots, k .\right.
$$

Algorithm 1 (Constructing the matrix P)

1. Let $P_{1}:=A_{k}$

2. For do $r=2,3, \ldots, k$ do

if $a_{k-r+1}=0$, then $\left(P_{r}\right)_{p q}:=P_{r-1}$ for $p=1,2, \ldots, n_{k-r+1}$

else $\left(P_{r}\right)_{p q}:=P_{r-1}$ if $p=q$

$$
\left(P_{r}\right)_{p q}:=\emptyset \quad \text { if } p \neq q
$$

where $\left(P_{r}\right)_{p q}:=P_{r-1}$ means that each sub-matrix $\left(P_{r}\right)_{p q}$ is the same as the 
principal sub-matrix $P_{r-1}$, and $\emptyset$ means the zero matrix with appropriate size and $P_{1}=A_{k}, P_{i}=A_{k-i+1} \otimes \ldots \otimes A_{k}$ for $i=1,2, \ldots, k$, and $\left(P_{i}\right)_{p q}$ denotes the $(p, q)^{t h}$ sub-matrix of $P_{i}$.

Note that in Theorem 3.2 each sub-matrix is the form of (4.1). So each sub-matrix of $X$ in Theorem 3.2 is constructed by using Algorithm 1.

For the matrix $X$ in Theorem 3.1, we have

$$
X X^{\prime}=\sum_{i=1}^{m} J_{n_{1}} \otimes J_{n_{2}} \otimes \ldots \otimes\left(I_{n_{i}}-\frac{1}{n_{i}} J_{n_{i}}\right) \otimes \ldots \otimes J_{n_{k}}
$$

where $I_{n_{0}}-\frac{1}{n_{0}} J_{n_{0}}$ is defined as $\frac{1}{a} J_{n_{i}}$ and $a=\left(\sum_{i=0}^{m} n_{i}^{-1}\right)^{-1}$.

Let $\mathrm{Q}$ a matrix defined to be of the form

$$
Q=B_{1} \otimes B_{2} \otimes \ldots \otimes B_{k}
$$

where each matrix $B_{i}$ is $I_{n_{i}}-\frac{1}{n_{i}} J_{n_{i}}$ either or $J_{n_{i}}$. Define $Q_{1}=B_{k}$, and $Q_{i}=B_{k-i+1} \otimes \ldots \otimes B_{k}$ for $i=2, \ldots, k$ then we have $Q_{i}=B_{k-i+1} \otimes Q_{i-1}$. We also define

$$
b_{i}= \begin{cases}1 & \text { if } B_{i}=I_{n_{i}}-\frac{1}{n_{i}} J_{n_{i}} \quad \text { for } i=1,2, \ldots, k . \\ 0 & \text { if } B_{i}=J_{n_{i}}\end{cases}
$$

Aim is to describe an algorithm for determining the matrix by a simple rule. Note that each matrix of sum in equation (4.2) is in form of matrix $Q$ for $j=0,1,2, \ldots, m$. Let $\left(Q_{i}\right)_{p q}$ denotes the $(p, q)^{t h}$ submatrix of $Q_{i}$.

Algorithm 2.a (Constructing the matrix $Q$ )

1. Let $Q_{1}:=B_{k}$

2. For $r=2,3, \ldots, k$ do

if $b_{k-r+1}=0$, then $\left(Q_{r}\right)_{p q}:=Q_{r-1}$ for all $p, q$

else $\quad\left(Q_{r}\right)_{p q}:=\left(1-\frac{1}{n_{k-r+1}}\right) Q_{r-1}$ if $p=q$

$$
\left(Q_{r}\right)_{p q}:=-\frac{1}{n_{k-r+1}} Q_{r-1} \text { if } p \neq q
$$

Let $\left(Q_{i}\right)_{p q}^{+}$denotes the $(p, q)^{t h}$ submatrix of $Q_{i}^{+}$.

$c_{i}=\left\{\begin{array}{ll}1 & \text { if } B_{i}^{+}=I_{n_{i}}-\frac{1}{n_{i}} J_{n_{i}} \\ 0 & \text { if } B_{i}^{+}=\frac{1}{n_{i}^{2}} J_{n_{i}}\end{array} \quad\right.$ for $i=1,2, \ldots, k$.

Note that $Q_{1}^{+}:=B_{k}^{+},\left(Q_{i}\right)^{+}:=\left(B_{k-i+1}\right)^{+} \otimes Q_{i-1}^{+}$and $\left(Q_{i}\right)^{+}=\left(B_{k-i+1} \otimes\right.$ $\left.\ldots \otimes B_{k}\right)^{+}$f or $i=2,3, \ldots, k$. Now, we will describe an algorithm for determining $Q^{+}$by a simple rule.

Algorithm 2.b (Constructing the matrix $Q^{+}$) 
1. Let $Q_{1}^{+}:=B_{k}^{+}$

2. For $r=2,3, \ldots, k$ do

if $c_{k-r+1}=0$, then $\left(Q_{r}\right)_{p q}^{+}:=\frac{1}{n_{k-r+1}^{2}} Q_{r-1}$ for all $p, q$

else $\left(Q_{r}\right)_{p q}^{+}:=\left(1-\frac{1}{n_{k-r+1}}\right) Q_{r-1}^{+}$if $p=q$

$$
\left(Q_{r}\right)_{p q}^{+}:=-\frac{1}{n_{k-r+1}} Q_{r-1}^{+} \quad \text { if } p \neq q
$$

where $B_{k}^{+}$is $I_{n_{k}}-\frac{1}{n_{k}} J_{n_{k}}$ either or $\frac{1}{n_{k}^{2}} J_{n_{k}}$. In Algorithm 2.a, $\left(Q_{r}\right)_{p q}:=Q_{r-1}$ means that each submatrix $\left(Q_{r}\right)_{p q}$ is the same as the principal submatrix $Q_{r-1}$, and in Algorithm 2.b, $\left(Q_{r}\right)_{p q}^{+}:=Q_{r-1}^{+}$means that each submatrix $\left(Q_{r}\right)_{p q}^{+}$is the same as the principal submatrix $Q_{r-1}^{+}$.

In Theorem 3.1 since $X^{\prime}=\left(P^{(0)}, P^{(1)}, \ldots, P^{(m)}\right)^{\prime}$ and $\left(X X^{\prime}\right)^{+}:=\sum_{j=0}^{m}\left(Q^{(j)}\right)^{+}$ and $X^{+}=X^{\prime}\left(X X^{\prime}\right)^{+}=\left(P^{(0)}, P^{(1)}, \ldots, P^{(m)}\right)^{\prime} \sum_{j=0}^{m}\left(Q^{(j)}\right)^{+}$

We get

$$
X=\left[\begin{array}{c}
\left(P^{(0)}\right)^{\prime}\left(Q^{(0)}\right)^{+} \\
\left(P^{(1)}\right)^{\prime}\left(Q^{(0)}\right)^{+}+\left(P^{(1)}\right)^{\prime}\left(Q^{(1)}\right)^{+} \\
\left(P^{(2)}\right)^{\prime}\left(Q^{(0)}\right)^{+}+\left(P^{(2)}\right)^{\prime}\left(Q^{(2)}\right)^{+} \\
\cdot \\
\cdot \\
\left(P^{(m)}\right)^{\prime}\left(Q^{(0)}\right)^{+}+\left(P^{(m)}\right)^{\prime}\left(Q^{(m)}\right)^{+}
\end{array}\right]
$$

where $P^{(0)}$ is the direct product of $\mathrm{k} j_{n_{r}}$ matrices and $\left(Q^{(0)}\right)^{+}$is the M-P inverse of the direct product of $\mathrm{k} J_{n_{r}}$ matrices and $\left(P^{(j)}\right)$ consist of the direct product of $\mathrm{k}$ matrices such that $j^{\text {th }}$ matrix is identity matrix and others are vectors with all elements equal to 1 , and $\left(Q^{(j)}\right)^{+}$consist of the direct product of $\mathrm{k}$ matrices such that $j^{\text {th }}$ matrix is $I_{n_{i}}-\frac{1}{n_{i}} J_{n_{i}}$ and others are $J_{n_{i}}$ for $j=1,2, \ldots, m$. It is easy to see that $X^{+}$is the same as given in (3.1).

Now, let $T=C_{1} \otimes C_{2} \otimes \ldots \otimes C_{k}$ and $T_{i}=C_{k-i+1} \otimes \ldots \otimes C_{k}$ for $i=1,2, \ldots, k$. Then $T_{1}=C_{k}$ and $T_{i+1}=C_{k-i} \otimes T_{i}$ where $C_{i}=I_{n_{i}}+J_{n_{i}}$. We can describe algorithms for determining the matrix $T$ and the matrix $T^{+}$respectively.

Algorithm 3. (Constructing the matrix $T$ or $X X^{\prime}$ in Theorem 3.2)

1. Let $T_{1}:=C_{k}$

2. For $r=2,3, \ldots, k$ do

$\left(T_{r}\right)_{p q}:=2 T_{r-1}$ if $p=q$

$\left(T_{r}\right)_{p q}:=T_{r-1}$ if $p \neq q$

Algorithm 4. (Constructing the matrix $T^{+}$or $\left(X X^{\prime}\right)^{+}$in Theorem 3.2)

1. Let $T_{1}^{+}:=C_{k}^{+}$

2. For $r=2,3, \ldots, k$ do

$\left(T_{r}\right)_{p q}^{+}:=\frac{n_{k-r+1}}{n_{k-r+1}+1} T_{r-1}^{+} \quad$ if $p=q$ 


$$
\left(T_{r}\right)_{p q}^{+}:=-\frac{1}{n_{k-r+1}+1} T_{r-1}^{+} \text {if } p \neq q
$$

where $C_{k}^{+}=I_{n_{k}}-\frac{1}{n_{k}+1} J_{n_{k}}$ and $\left(T_{i+1}\right)^{+}=C_{k-i}^{+} \otimes T_{i}^{+}$, and $\left(T_{r}\right)_{p q}^{+}:=T_{r-1}^{+}$means that each sub-matrix $\left(T_{r}\right)_{p q}^{+}$is the same as the principal sub-matrix $\left(T_{r-1}\right)^{+}$ and $p, q=1,2, \ldots, n_{k-r+1}$.

Example 3 Let $k=3$ and $T$ be a matrix of the form

$$
T=C_{1} \otimes C_{2} \otimes C_{3}=\left(I_{n_{1}}+J_{n_{1}}\right) \otimes\left(I_{n_{2}}+J_{n_{2}}\right) \otimes\left(I_{n_{3}}+J_{n_{3}}\right)
$$

we can construct the matrix $T$ by using Algorithm 3 as follows :

1. Let $T_{1}=C_{3}=I_{n_{3}}+J_{n_{3}}$

2. For $r=2$ we obtain

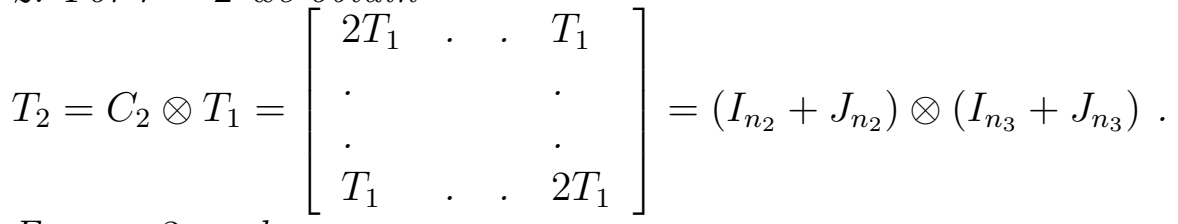

For $r=3$ we have

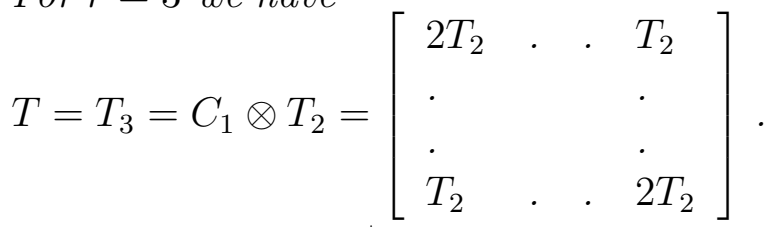

we also construct $T^{+}$by using Algorithm 4 as follows :

1. Let $T_{1}^{+}:=C_{3}^{+}=I_{n_{3}}-\frac{1}{n_{3}+1} J_{n_{3}}$

2. For $r=2$ we obtain

$$
T_{2}^{+}=C_{2}^{+} \otimes T_{1}^{+}=\left[\begin{array}{lll}
\frac{n_{2}}{n_{2}+1} T_{1}^{+} & \cdot & -\frac{1}{n_{2}+1} T_{1}^{+} \\
\cdot & \cdot \\
\cdot-\frac{1}{n_{2}+1} T_{1}^{+} & \cdot & \cdot \\
\frac{n_{2}}{n_{2}+1} T_{1}^{+}
\end{array}\right]
$$

and for $r=3$ it is end of the loop and we get

$$
T^{+}=T_{3}^{+}=C_{1}^{+} \otimes T_{2}^{+}=\left[\begin{array}{lll}
\frac{n_{1}}{n_{1}+1} T_{2}^{+} & \cdot & -\frac{1}{n_{1}+1} T_{2}^{+} \\
\cdot & \cdot \\
\cdot-\frac{1}{n_{1}+1} T_{2}^{+} & \cdot & \cdot \frac{n_{1}}{n_{1}+1} T_{2}^{+}
\end{array}\right]=\left(X X^{\prime}\right)^{+} \text {. }
$$

Now we will illustrate computations of M-P inverse of matrix $X$ in Theorem 3.2. Let $P_{i}$ be a matrix consisting of the direct product of i $I_{n_{r}}$ identity matrices and $(\mathrm{k}-\mathrm{i}) j_{n_{r}}^{\prime}$ matrices for $i=0,1,2, \ldots, k$. It is obvious that each $P_{i}$ has $\left(\begin{array}{c}k \\ i\end{array}\right)$ block matrices, and each block of $P_{i}$ can be constructed by replacing $j_{n_{r}}$ by $j_{n_{r}}^{\prime}$ in Algorithm 1. For example $P_{1}$ has k block matrices. Each block is consist of direct product of $\mathrm{k}$ matrices which any one is matrix $I_{n_{r}}$ and other ones are matrices $j_{n_{r}}^{\prime}$. The matrix $P_{i}$ is transpose of the matrix $X_{i}$ which is defined in 
Theorem 3.2. To compute M-P inverse of matrix $X$ in Theorem 3.2 first we find $\left(X X^{\prime}\right)^{+}$by using Algorithm 4 then we have

$$
X^{+}=\left[\left(\tilde{P}_{0}\right)^{\prime},\left(\tilde{P}_{1}\right)^{\prime}, \ldots,\left(\tilde{P}_{k-1}\right)^{\prime},\left(\tilde{P}_{k}\right)^{\prime}\right]^{\prime}
$$

where $\tilde{P}_{i}^{\prime}=P_{i}\left(X X^{\prime}\right)^{+}$. It is clear that $P_{0}\left(X X^{\prime}\right)^{+}=\left(\prod_{j=1}^{k} \frac{1}{n_{j}+1}\right) j_{n_{1}}^{\prime} \otimes j_{n_{2}}^{\prime} \otimes$ $\ldots \otimes j_{n_{k}}^{\prime}$ and the matrix $P_{1}\left(X X^{\prime}\right)^{+}$has $\mathrm{k}$ block matrices and each sub-block of $P_{1}\left(X X^{\prime}\right)^{+}$is

$$
\left(\prod_{j=1}^{k} \frac{1}{n_{j}+1}\right) j_{n_{1}}^{\prime} \otimes j_{n_{2}}^{\prime} \otimes\left(I_{n_{i}}-\frac{1}{n_{i}+1} J_{n_{i}}\right) \otimes j_{n_{i+1}}^{\prime} \otimes \ldots \otimes j_{n_{k}}^{\prime}
$$

for $i=1,2, \ldots, k . P_{2}\left(X X^{\prime}\right)^{+}$has $\left(\begin{array}{c}k \\ 2\end{array}\right)$ block matrices and each subblock of $P_{2}\left(X X^{\prime}\right)^{+}$is

$v j_{n_{1}}^{\prime} \otimes j_{n_{2}}^{\prime} \otimes\left(I_{n_{i}}-\frac{1}{n_{i}+1} J_{n_{i}}\right) \otimes j_{n_{i+1}}^{\prime} \otimes \ldots \otimes\left(I_{n_{r}}-\frac{1}{n_{r}+1} J_{n_{r}}\right) \otimes j_{n_{r+1}}^{\prime} \otimes \ldots \otimes j_{n_{k}}^{\prime}$

for $i=1,2, \ldots, k ; r=1,2, \ldots, k$ and $i<r$, where $v=\prod_{j=1, j \neq i, j \neq r}^{k} \frac{1}{n_{j}+1}$. Similarly, we can construct others $\tilde{P}_{i}^{\prime}=P_{i}\left(X X^{\prime}\right)^{+}$matrices.

\section{References}

[1] A. Albert, Regression and the Moore-Penrose Pseudo Inverse, Academic Press, New York (1972).

[2] A. Ben-Israel and T. Greville, Generalized Inverses: Theory and Applications, Wiley, New York (1974).

[3] L. Boullion and P.L. Odell, Generalized Inverse Matrices, Wiley, New York (1971).

[4] F:A. Graybill, Matrices with Applications in Statistics, Wadsworth Int. Group. Belmont California (1983).

[5] D.A. Harville, Matrix Algebra From a Statistician's Perspective, Springer - Verlag, New York (1997).

[6] B.C. Kim and J.T. Lee, The Moore-Penrose inverse for the classificatory models, Journal of the Korean Statistical Society, Vol.15(1), (1986), 46 61. 
[7] R. Penrose, A generalized inverse for matrices, Proc. Cambridge Philos. Soc. 51, (1955), 406 - 413.

[8] R. Pringle and A. Rayner, Generalized Inverse Matrices with Application in Statistics, Hafner, New York (1971).

[9] C.R. Rao and S.K. Mitra, Generalized Inverse of Matrices and its Applications, Wiley, New York (1971).

[10] S.R. Searle, Matrix Algebra Useful for Statistics, Wiley, New York (1982).

[11] J.R. Schott, Matrix Analysis for Statistics, Wiley, New York (1997).

[12] H. Sunwoo, Simple algorithms about Kronecker product in the linear model, Linear Algebra and Its Applications, 237/238, (1996), 351 - 358.

Received: August 21, 2005 